\title{
Mapping QTLs using High-Density SNPs Genotyped by Sequencing Reveals Novel Potential Regions Underlying Maize Root Morphological Traits at Seedling Stage
}

\author{
Abdourazak Alio Moussa ${ }^{1 *}$, Ajmal Mandozai ${ }^{1}$ Jing Qu ${ }^{1}$, Yukun Jin ${ }^{1}$, Qi Zhang ${ }^{1}$, Mahmoud Gamal Abd El-Rahim ${ }^{2}$, \\ Gulaqa Anwari ${ }^{1}$, Ahmed Sharaf ${ }^{2}$ and Piwu Wang ${ }^{1}$ \\ ${ }^{1}$ College of Agronomy, Plant Biotechnology Center, Jilin Agricultural University, Changchun, 130118, Jilin, P. R. China \\ ${ }^{2}$ College of Resources and Environmental Sciences, Jilin Agricultural University, Changchun, 130118, Jilin, P. R. China \\ *For correspondence: abdoulrazakalio@gmail.com \\ Received 11 September 2020; Accepted 05 January 2021; Published 25 March 2021
}

\begin{abstract}
Maize (Zea mays L.) root system plays a crucial role in plant fixation and the acquisition of nutrients and water essential for growth and development. Herein, 179 recombinant inbred lines (RILs) obtained from a cross between P014 $\times$ E1312 were genotyped via genotyping-by-sequencing (GBS) and phenotyped for root related-traits at 5 and 15 days after germination (dag) under controlled conditions. Quantitative trait locus (QTL) mapping based on high-density GBS-SNPs bin map was performed, and an overall number of 14 QTLs with a phenotypic variance explained (PVE) ranging from 1.78 to $16.05 \%$ were identified. The QTL co-localization was detected at each of the two time-points, and one major QTL region on chromosome 4 was found to be significantly associated with multiple traits, including root projected area (PRA), root surface area (SUA), shoot dry weight (SDW), and total plant biomass (TPB). Compared to previous root-related studies, QTLs located in chromosomal bins 2.09 (qROT $\left.\mathrm{qd}^{-2-1}\right), 4.05$ (qPRA $\left._{5 \mathrm{~d}}-4-1, \mathrm{qSUA}_{5 \mathrm{~d}}-4-1, \mathrm{qSDW}_{15 \mathrm{~d}}-4-1, \mathrm{qTPB}_{15 \mathrm{~d}}-4-1\right), 7.06$ (qTRL $\left._{15 \mathrm{~d}}-7-1\right)$, and 8.09 (qSDW $_{5 \mathrm{~d}}-8-1$ ) were found to be novel. Two candidate genes GRMZM2G109056 and GRMZM2G053458, associated with root dry weight trait on chromosome 1, were verified for expression level, and the results showed significantly different expression levels between the two outer parental accessions in primary roots at all evaluated time-points. Thus, the identified loci and genes could play an important role in maize molecular breeding for high yielding varieties. C 2021 Friends Science Publishers
\end{abstract}

Keywords: Genotyping-by-sequencing; High-density SNPs bin-map; Maize seedlings; QTL; Root morphological traits, qRT-PCR

\section{Introduction}

Maize (Zea mays L.) root system plays a critical role in plant anchorage and acquisition of water and nutrients (Song et al. 2016). Changes in the root architecture of maize strongly affect the yield (Hammer et al. 2009). Previous studies have shown that high-yielding maize varieties displayed propitious root architecture that can provide strong supplies of water and nutrients, resulting in increased grain yields (Hammer et al. 2009; Abdel-Ghani et al. 2013). However, notwithstanding their notorious involvement in plant development, agricultural performance, and competition in the wild, roots in plant genetics remain an under-explored frontier. The visualization and measurement of root structures and their growth are much challenging than the characterization of aboveground parts of the plant and is often simply avoided (Bray and Topp 2018). The majority of maize root investigations have focused on their anatomy, physiology, growth, development, and soil interactions but far less is known about the genetics that regulates root quantitative traits (Bray and Topp 2018). To date, quantitative variation has been proposed for root architecture to promote trait optimization in various environments through a systematic "fine-tuning" of many loci (Gifford et al. 2013; Rosas et al. 2013). Nevertheless, this concept has not been well tested in crops, mainly because few genes that regulate root traits have been identified. The main challenge in the genetic analysis of root characteristics is the need for reliable and high-throughput phenotypic assessment methods that can provide a proxy for field performance since measuring root characteristics under open-field conditions can be extremely challenging (Iannucci et al. 2017). This is especially the case for genetic studies involving very large samples. Therefore, different growth strategies are being used under laboratory, greenhouse, as well as field conditions, and soil-less growth media is the most commonly-used growth system (Wasaya et al. 2018). Plant growing methods that nearly mimic the soil media have been reported to be more stable in mineral elements and environmental factors, easier to operate, and

To cite this paper: Moussa AA, A Mandozai, J Qu, Y Jin, Q Zhang, MGA El-Rahim, G Anwari, A Sharaf, P Wang (2021). Mapping QTLs using high-density SNPs genotyped by sequencing reveals novel potential regions underlying maize root morphological traits at seedling stage. Intl J Agric Biol 25:904-914 
more appropriate for root morphological traits phenotyping in maize ( $\mathrm{Ju}$ et al. 2018). Therefore, to offer a better and robust tool for plant behavior prediction under field conditions, various experimental growing systems with soilbased substrates have been adopted (Zhu et al. 2005; Laperche et al. 2006; Ren et al. 2012; Liu et al. 2013). The root phenotypic data acquisition is now becoming cheaper, quicker, and more effective, mainly due to rapid progress in digitally automatic image analysis (Galkovskyi et al. 2012; Pierret et al. 2013; Pace et al. 2014; Das et al. 2015; RellánÁlvarez et al. 2015; Symonova et al. 2015). Today, image processing approaches have been widely used as reliable and less time-consuming root phenotyping techniques and have become accessible through various softwares. Recently, several studies have attempted to examine the genetic basis of maize root system architectures in both field (Zaidi et al. 2016; Gu et al. 2017; Zhang et al. 2018) and controlled conditions (Pace et al. 2015a, b; Zurek et al. 2015; Song et al. 2016; Liu et al. 2017; Sanchez et al. 2018). However, most of those studies used relatively short time-point intervals and low-density marker genetic maps, resulting in large inter-marker intervals (Song et al. 2016). The full realization of the importance of root architecture for crop improvement will require, therefore, a more comprehensive understanding of the particular genetic loci involved in the variability of quantitative root traits (Price et al. 2007; Hochholdinger and Tuberosa 2009; Lynch 2013). Thus, we hypothesized that longer time-point intervals combined with high-density Single Nucleotide Polymorphism (SNP) bin-map would more efficiently and accurately estimate Quantitative Trait Loci (QTLs).

The present study aimed to (i) construct a high-density GBS-SNPs genetic bin-map for a RIL population obtained from a cross between $\mathrm{P} 014 \times$ E1312; (ii) map novel potential chromosomal regions underlying maize root morphological traits at 5 and 15 days after germination; and (iii) detect candidate genes associated with root related-traits.

\section{Materials and Methods}

\section{Genetic material and growth conditions}

The two parental maize lines used in this study, P014, E1312, and their 179 offspring were used as genetic material for the analysis of root characteristics. The RIL family comprising 179 inbred lines was developed by a cross between the maternal line P014 and the male parent E1312 and continuous inbreeding for 9 generations. Genetically pure seeds were obtained from the Jilin Agricultural University's experimental field in 2018.

\section{Plant materials and growth conditions}

The experiments were laid out using a completely randomized block (CRD) in growth chambers under a photoperiod of $14 / 10 \mathrm{~h}$ at a temperature of $25 / 22^{\circ} \mathrm{C}$ and relative humidity of $70 / 80 \%$ (light/darkness). The light intensity was set at $200 \mu \mathrm{mol}$ photons $\mathrm{m}^{-2} \mathrm{~s}^{-1}$. Two independent growth chamber trials were completed in April and July 2019. Seedlings were planted in polyvinyl chloride (PVC) pipes sealed at the bottom with 4 manually pierced holes for drainage. The dimensions of the PVC pipe were 26 $\mathrm{cm}$ and $9 \mathrm{~cm}$ in height and bottom diameter. The PVC pots were filled with a mixture of sandy soil and vermiculite (2:1 ratio). For data collection, each pot consisted of three (3) seedlings was considered an experimental unit.

\section{Root phenotyping}

At each indicated time-point (5 and 15dag), seedlings were taken off from the growth chamber and harvested for root traits analysis. To eliminate soil residuals, the samples were carefully removed from the pots and washed concisely with water. In each trial, 3 seedlings per line were examined simultaneously at each specified time-point. All the collected traits are listed in Table 1. To generate high-resolution images, each root sample was put on a scanner (Perfection V800 Epson, resolution of 12800 dots per inch (dpi: 5039.37 dots per $\mathrm{cm}$ )) and using DJ-GXG02 software (www. Dianjiangtech.com), the root images were then processed. If root scanning could not be completed in a single day, seedlings were preserved by submerging the roots in $30 \%$ ethanol and storing them in a cold room (about $4{ }^{\circ} \mathrm{C}$ ) to prevent further growth (Sanchez et al. 2018). Root and shoot dry weights were measured after drying in an oven dryer set at $75^{\circ} \mathrm{C}$ for at least $48 \mathrm{~h}$ until constant weight. For that purpose, an electronic scale was used. A total of 10 traits including five (4) manually measured (root dry weight, shoot dry weight, root to shoot dry weight ratio, total plant biomass) and five (5) electronically recorded via DJ-GXG02 (total root length, surface area, projected area, average root diameter, and root tips) were collected (Table 1).

\section{Root traits statistical analysis}

All statistical analyses were performed using Minitab17 software (Minitab Inc., State College, P.A., U.S.A.). For each root related trait in the two parental lines and RIL population, descriptive statistics including mean, standard deviation, maximum and minimum were calculated. Analysis of variance (ANOVA) using the Fit General Linear Model was carried out to compare the two parental maize inbred lines for the different morphological traits assessed at the respective time-points. As well, broad-sense heritability $\left(h^{2}\right)$ was determined on an entry mean basis as described by Pace et al. (2015a, b). Normal distribution and Pearson coefficients of correlation among traits in the RIL family were also generated.

\section{Genotyping, QTL mapping, and linkage map analysis}

The RIL population was genotyped by sequencing (GBS) (www.broadinstitute.org/gatk), and MSTmap software 
Table 1: Collected root related traits initials and illustrations

\begin{tabular}{lll}
\hline Trait Name & Abbreviations & Trait description \\
\hline Root dry weight & RDW & Total root dry weight of the plant in gram \\
Shoot dry weight & SDW & Total shoot dry weight the plant in gram \\
Root dry weight/Shoot dry weight & RDW/SDW & Root to shoot dry weight ratio in gram \\
Total plant biomass & TPB & Total root dry weight + Total shoot dry weight in gram \\
Total root length & TRL & Cumulative length of all the roots in $\mathrm{cm}^{2}$ \\
Projected area & PRA & Whole root system projected area in $\mathrm{cm}^{2}$ \\
Surface area & SUA & Whole root system surface area in $\mathrm{cm}^{2}$ \\
Average root diameter & ARD & The average diameter of the entire root system mm \\
Root tips & ROT & Total number of all the root tips \\
\hline
\end{tabular}

(Wu et al. 2008) was used for linkage analysis of marker data. Version 4.1 of QTL IciMapping software was used to perform QTL mapping (Wang et al. 2016). With reference to Meng et al. (2015) and based on genotypic data of the 179 RILs with a final total of 4235 high-quality SNP markers spanned on all chromosomes, linkage map analysis was undertaken using automatic parameter settings with the required data. Using the Kosambi mapping function, recombination frequencies and pairwise distance were converted in centimorgans $(\mathrm{cM})$. The relative segregation ratio of each marker and its relative deviation from the expected ratio were determined via the squared chi test. Briefly, the markers were grouped at a LOD of $\geq 3.0$, ordered, rippled then outputted to construct the linkage map which covered a total length of $1514.57 \mathrm{cM}$ distributed on the 10 linkage groups. QTL analysis was performed following inclusive composite interval mapping for additive QTL (ICIM-ADD), and a 1000 permutation test at $95 \%$ confidence level was used to define QTL logarithm of odds (LOD) scores threshold. The walking speed was $1.0 \mathrm{cM}$ and the size of the windows was $5.0 \mathrm{cM}$. A LOD threshold peak score value of $\geq 2.5$ was set to declare a significant QTL, which is commonly used in maize QTL mapping (Li et al. 2014; Song et al. 2016). QTL additive effects and phenotypic variance explained (PVE) were also analyzed.

\section{Gene annotation and expression analysis}

With reference to the B73 genome, MaizeGDB (http://www.maizegdb.org/) and Gramene (https://www.gramene.org/) databases were used for predicting functional annotations of the candidate genes. Two candidate genes GRMZM2G109056 and GRMZM2G053458, with known functions in maize root growth (Stelpflug et al. 2016; Hoopes et al. 2019) were tested for expression analysis via quantitative real-time PCR (qRT-PCR) using primary root samples from the two parental accessions ( P014 and E1312) at the two evaluated time-points (5dag and 15dag). The total RNA was mined using an RNA kit (TIANGEN, China), and cDNA was synthesized using a standard protocol based on the Prime ScriptTM RT Reagent Kit (TaKaRa, Japan). qRT-PCR primers were designed using Primer Premier 5.0 software, and Leunig (GenBank accession: GRMZM2G425377_T01) was selected as the reference gene (Manoli et al. 2012). All primer sequences were presented in Table 2. qRT-PCR trials in triplicates were carried out in a system of $20 \mu \mathrm{L}$, consisting of $2 \mu \mathrm{L} \mathrm{cDNA}, 1 \mu \mathrm{L}$ of every single primer $(\mu \mathrm{M})$, and $10 \mu \mathrm{L}$ qPCR Master mix using a Stratagene Mx3000P instrument. The relative level of expression of the two indicated genes was determined using $2^{-\triangle \Delta C T}$ method (Livak and Schmittgen 2001).

\section{Results}

\section{Root morphological traits phenotypic variability}

The seedlings were developed in a 2:1 ratio mixture of sandy soil and vermiculite in a growth chamber for root trait measurements. The results related to morphological performances of root and shoot traits display large variations both in parents and their offspring (Table 3). Apparent differences about roots between the two parents of the 179 RILs, P014 and E1312, were observed at 5dag and 15dag. P014 possessed a longer root system than E1312 from 5dag. At 15dag, P014 compare to E1312 showed a ticker root system with a larger and longer number of lateral roots present on the primary and seminal roots. The inbred line P014 was therefore superior in phenotype because of its larger number of roots especially at the later time point (15 dag). We also evaluated the architectural differences among the two root systems of the parental lines about the nine measured traits. Results from the study of the parental phenotypic data showed gradual increases in eight of the nine root and shoot morphological traits across the two time-points. In contrast, among the other traits, ARD and RDW/SDW, progressively dropped, due likely to the continual emergence of new leaves and roots by time. This result shows the instantaneous nature of the development of the two parental root systems overtime which confirms the pertinence of the two experimental time-points selected for root traits assessment in this study. Analysis of variance related to the morphological differences between the root systems of the two parental lines reveals highly significant variations $(P<0.05 ; P<0.01 ; P<0.001)$ in the measured root related traits from the parental inbred lines (Table 2). Comparatively, P014 showed higher values for, RDW, TPB, TRL, PRA, SUA, and ROT than E1312 across both time-points (Table 3). This supports the pertinence as well as the relevance of using the two parental lines in this current study. For illustration, at 5dag, RDW, TPB, TRL, PRA, SUA and ROT in P014 versus E1312 were $0.04 \mathrm{~g}$, 
Potential Regions Underlying Maize Root Morphological Traits / Intl J Agric Biol, Vol 25, No 4, 2021

Table 2: Oligonucleotide primers used for qRT-PCR

\begin{tabular}{ll}
\hline GRMZM2G109056-forward & 5'ATGTTCTGGCACGGGGTCGCGGA3' \\
\hline GRMZM2G109056-reverse & 5'CGAGGAGCGAGGCGTTGAAGTCG3' \\
GRMZM2G053458-forward & 5'ATGCCAGACCACGGGCACGGGGT 3' \\
GRMZM2G053458-reverse & 5'TGGAACTGCCCGGACGGACACCC3' \\
Leunig-forward & 5'TCCAGTGCTACAGGGAAGGT3' \\
Leunig-reverse & 5'GTTAGTTCTTGAGCCCACGC3' \\
\hline
\end{tabular}

Table 3: Root parameters performances in both parental and RIL lines

\begin{tabular}{|c|c|c|c|c|c|c|c|c|}
\hline Traits & Time $^{\mathrm{a}}$ & E1312 & $\mathrm{P} 014$ & Sig. & RILs & & & $\mathrm{h}^{2}(\%) \mathrm{c}$ \\
\hline & (dag) & Mean \pm SD & Mean \pm SD & Level $^{b}$ & Mean \pm SD & Min & Max & \\
\hline \multirow[t]{2}{*}{$\mathrm{SDW}(\mathrm{g})$} & 5 & $0.03 \pm 0.00$ & $0.04 \pm 0.01$ & $*$ & $0.05 \pm 0.02$ & 0.01 & 0.11 & 45.4 \\
\hline & 15 & $0.10 \pm 0.02$ & $0.11 \pm 0.01$ & ns & $0.17 \pm 0.07$ & 0.03 & 0.47 & 49.7 \\
\hline RDW (g) & 15 & $0.03 \pm 0.01$ & $0.06 \pm 0.01$ & * & $0.05 \pm 0.03$ & 0.01 & 0.22 & 64.1 \\
\hline \multirow[t]{2}{*}{ TPB (g) } & 5 & $0.04 \pm 0.00$ & $0.08 \pm 0.01$ & $* *$ & $0.08 \pm 0.04$ & 0.01 & 0.21 & 58.8 \\
\hline & 15 & $0.13 \pm 0.02$ & $0.17 \pm 0.01$ & * & $0.22 \pm 0.09$ & 0.04 & 0.63 & 55.3 \\
\hline \multirow[t]{2}{*}{ RDW/SDW } & 5 & $0.38 \pm 0.04$ & $0.87 \pm 0.34$ & ns & $0.90 \pm 1.56$ & 0.09 & 17.00 & 44.9 \\
\hline & 15 & $0.32 \pm 0.12$ & $0.50 \pm 0.05$ & ns & $0.33 \pm 0.21$ & 0.05 & 2.33 & 39.7 \\
\hline \multirow[t]{2}{*}{$\operatorname{TRL}(\mathrm{cm})$} & 5 & $36.86 \pm 2.43$ & $70.45 \pm 6.28$ & $* *$ & $57.54 \pm 35.03$ & 1.81 & 443.89 & 40.4 \\
\hline & 15 & $107.86 \pm 6.53$ & $216.63 \pm 2.55$ & $* * *$ & $134.09 \pm 80.75$ & 5.17 & 597.46 & 53.4 \\
\hline \multirow[t]{2}{*}{$\operatorname{PRA}\left(\mathrm{cm}^{2}\right)$} & 5 & $5.12 \pm 0.69$ & $9.25 \pm 1.00$ & $* *$ & $6.84 \pm 9.55$ & 0.86 & 203.26 & 7.6 \\
\hline & 15 & $11.31 \pm 0.69$ & $22.68 \pm 0.55$ & $* * *$ & $14.00 \pm 9.37$ & 1.25 & 61.55 & 54.6 \\
\hline \multirow[t]{2}{*}{$\operatorname{SUA}\left(\mathrm{cm}^{2}\right)$} & 5 & $16.09 \pm 2.17$ & $28.19 \pm 4.20$ & * & $21.50 \pm 30.01$ & 2.69 & 638.57 & 7.6 \\
\hline & 15 & $35.71 \pm 1.86$ & $72.96 \pm 4.58$ & $* * *$ & $43.98 \pm 29.45$ & 3.92 & 193.36 & 54.6 \\
\hline $\mathrm{ARD}(\mathrm{mm})$ & 5 & $1.56 \pm 0.31$ & $1.32 \pm 0.09$ & ns & $1.21 \pm 0.50$ & 0.58 & 5.56 & 22.7 \\
\hline \multirow[t]{2}{*}{ ROT } & 5 & $241.33 \pm 14.05$ & $381.33 \pm 17.50$ & $* * *$ & $362.48 \pm 212.05$ & 14.00 & 2223.00 & 46.8 \\
\hline & 15 & $641.67 \pm 8.50$ & $1175.33 \pm 7.09$ & $* * *$ & $821.47 \pm 625.95$ & 69.00 & 6969.00 & 71.9 \\
\hline
\end{tabular}

${ }^{\mathrm{a}}$ Time points for root traits assessment: 5 and 15 dag (days after germination). ${ }^{\mathrm{b}}$ Probability level of significance via student-test with, ns: no significant difference; $*$ : significantly different at $P<0.05$; ** : significantly different at $P<0.01$ and $* * *$ : significantly different at $P<0.001$. ${ }^{\mathrm{c}}$ Heritability estimates (broad sense). Root dry weight (RDW), shoot dry weight (SDW), total plant biomass (TPB), root to shoot dry weight (RDW/SDW), total root length (TRL), projected area (PRA), surface area (SUA), average root diameter (ARD), and root tips (ROT)

$0.08 \mathrm{~g}, 70.45 \mathrm{~cm}, 9.25 \mathrm{~cm}^{2}, 28.19 \mathrm{~cm}^{2}$, and $381 ; 0.01 \mathrm{~g}, 0.04$ $\mathrm{g}, 36.86 \mathrm{~cm}, 5.12 \mathrm{~cm}^{2}, 16.09 \mathrm{~cm}^{2}$, and 241 , respectively (Table 3). At 15 dag, P014 performed for the traits RDW, TPB, TRL, PRA, SUA, and ROT, $0.06 \mathrm{~g}, 0.17 \mathrm{~g}, 216.63$ $\mathrm{cm}, 22.68 \mathrm{~cm}^{2}, 72.96 \mathrm{~cm}^{2}$ and 1175.33 , respectively, while E1312 presented respectively $0.03 \mathrm{~g}, 0.13 \mathrm{~g}, 107.86 \mathrm{~cm}$, $11.31 \mathrm{~cm}^{2}, 35.71 \mathrm{~cm}^{2}$ and 641.67 for the same traits (Table $3)$. Given together, these findings indicate that the two parents of the RIL population exhibited substantial differences in most of the evaluated root and shoot features at both time-points.

Subsequently, we examined the distribution of the root related-traits in the 179 RILs. A large spectrum of variation was detected for each trait (Table 3). The heritability values ranged between 7.6 and $71.9 \%$, and the highest value was observed from ROT with $71.9 \%$, while SUA and PRA with $7.6 \%$ showed the lowest heritability (Table 3 ). For most of the traits evaluated, heritability estimates were moderate (> $41 \%$ ). Similar correlation patterns were detected at all two time-points. At $15 \mathrm{dag}$, a greater extent of significant correlations was observed comparing to 5 dag (Table 4). In terms of comparison, the correlation coefficients between RDW, TPB, TRL, PRA, SUA, and ROT were similar at 5 dag and 15 dag, but more strongly in 15 days old seedlings (Table 4). In regards of all traits, SUA and PRA exhibited the strongest significant correlations $(r=0.990)$ while RDW and SHL showed the lowest ones both at 5 dag $(r=0.157)$ and 15 dag $(r=0.194)$. TRL is correlated with all other traits at both time-points except ARD and RDW/SDW at 5 dag and ARD at 15 dag (Table 4). For example, strong to moderate significant correlations at 15 dag were obtained among TRL and PRA $(r=0.945)$, TRL and SUA $(r=$ 0.945), TRL and ROT ( $r=0.883)$, TRL and RDW $(r=$ $0.761)$, and TRL and TPB $(r=0.707)$ (Table $3 ; P<0.01)$. Interestingly, at $15 \mathrm{dag} \mathrm{RDW}$ is significantly $(P<0.05 ; P<$ $0.01)$ linked positively to all traits excluding ARD (Table 4$)$. The high correlation between ROT and RDW at 15dag ( $r=$ 0.600 ) confirmed the predominant contribution of root tip number to RDW, TPB and TRL. ARD is weakly correlated $(r=0.218-0.350)$ with only SDW, TPB, SUA, and PRA, in seedlings at $15 \mathrm{dag}$ (Table 4$)$.

\section{High-quality genetic linkage map}

With reference to the B73 genome, an initial total of 7275986 SNPs was obtained using GATK-samtools software (www.broadinstitute.org/gatk). After screening and filtering out the low-quality SNPs according to the following criteria: (i) SNPs sequencing depth for parents $<6$ fold, (ii) SNPs coverage for offsprings' number $<60 \%$, and (iii) SNPs displaying a highly significant $(P<0.01)$ segregation ratio, a panel of 63990 SNPs were obtained for linkage mapping. With the removal of unlinked and co-segregated SNP markers, we established the bin-map with 4235 high-quality 
Moussa et al. / Intl J Agric Biol, Vol 25, No 4, 2021

Table 4: Correlation coefficients of Pearson for root and shoot related traits in the 179 RILs at each time-point

\begin{tabular}{llllllllll}
\hline & SDW & RDW & TPB & RDW/SDW & TRL & PRA & SUA & ARD & ROT \\
\hline SDW & 1 & 0.138 & $0.635^{* *}$ & $-0.296^{* *}$ & $0.489^{* *}$ & $0.292^{* *}$ & $0.292^{* *}$ & -0.127 & $0.401^{* *}$ \\
RDW & $0.675^{* *}$ & 1 & $0.838^{* *}$ & $0.738^{* *}$ & $0.312^{* *}$ & $0.215^{* *}$ & $0.214^{* *}$ & $0.159^{*}$ & $0.287^{* *}$ \\
TPB & $0.959^{* *}$ & $0.853^{* *}$ & 1 & $0.416^{* *}$ & $0.509^{* *}$ & $0.326^{* *}$ & $0.326^{* *}$ & 0.045 & $0.441^{* *}$ \\
RDW/SDW & -0.018 & $0.628^{* *}$ & $0.226^{* *}$ & 1 & -0.010 & 0.010 & 0.010 & $0.200^{* *}$ & 0.062 \\
TRL & $0.594^{* *}$ & $0.761^{* *}$ & $0.707^{* *}$ & $0.380^{* *}$ & 1 & $0.770^{* *}$ & $0.771^{* *}$ & -0.092 & $0.834^{* *}$ \\
PRA & $0.652^{* *}$ & $0.766^{* *}$ & $0.749^{* *}$ & $0.322^{* *}$ & $0.945^{* *}$ & 1 & $0.990^{* *}$ & $0.292^{* *}$ & $0.628^{* *}$ \\
SUA & $0.652^{* *}$ & $0.766^{* *}$ & $0.749^{* *}$ & $0.322^{* *}$ & $0.945^{* *}$ & $0.990^{* *}$ & 1 & $0.292^{* *}$ & $0.628^{* *}$ \\
ARD & $0.242^{* *}$ & 0.132 & $0.218^{* *}$ & -0.109 & 0.078 & $0.350^{* *}$ & $0.350^{* *}$ & 1 & 0.063 \\
ROT & $0.364^{* *}$ & $0.600^{* *}$ & $0.485^{* *}$ & $0.381^{* *}$ & $0.883^{* *}$ & $0.822^{* *}$ & $0.822^{* *}$ & 0.076 & 1 \\
\hline
\end{tabular}

Correlation coefficients at $5 \mathrm{dag}$ (upper right) and $15 \mathrm{dag}$ (lower left). The symbol $*$ and $* *$ indicate respectively, significance at $P<0.05$ and at $P<0.01$. Root dry weight (RDW), shoot dry weight (SDW), total plant biomass (TPB), root to shoot dry weight (RDW/SDW), total root length (TRL), projected area (PRA), surface area (SUA), average root diameter (ARD), and root tips (ROT)

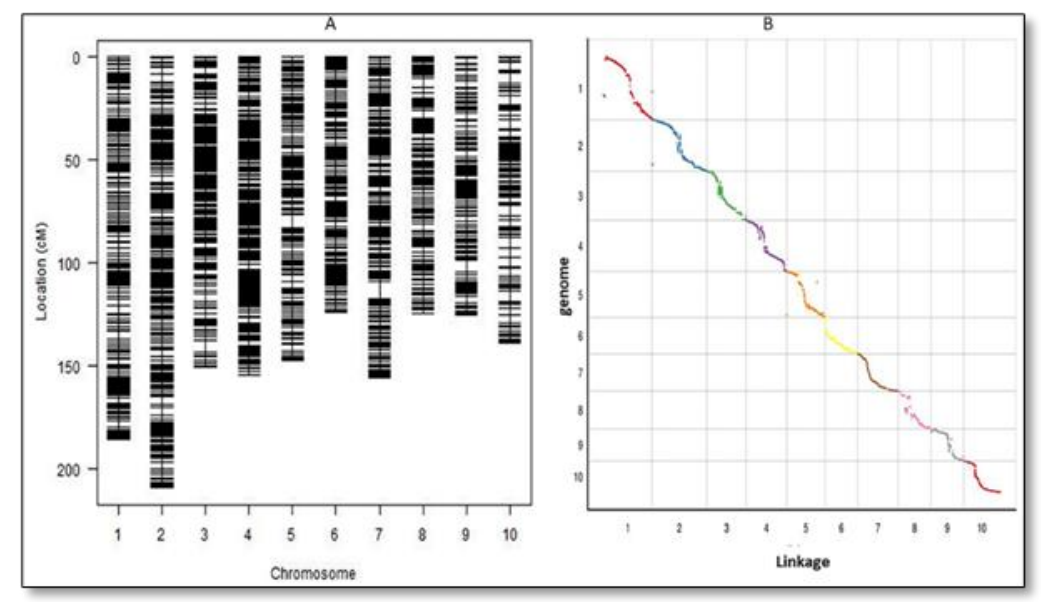

Fig. 1: P014 × E1312 RIL high-density GBS-SNPs genetic bin-map (A) and consistency map (B)

SNP markers spanned along 10 chromosomes (linkage groups). The generated map covered a total genetic length of $1514.57 \mathrm{cM}$, and no evident issues with the map were detected (Table 5, Fig. 1). The average number of markers per linkage group and the distance between successive markers were 423.5 and $0.38 \mathrm{cM}$, respectively (Table 5). Chromosome 4 contained the highest number of markers with 635 markers spanning $154.16 \mathrm{cM}$ (the highest density) while chromosome 8 contained the lowest number with 285 markers spanning $124.63 \mathrm{cM}$. The lowest marker density consisted of 389 markers spanning $185.41 \mathrm{cM}$ on chromosome 1 (Table 5). Among the 10 chromosomes, the most extented genetic length was $209.19 \mathrm{cM}$ containing 477 bin markers for chromosome 2 . In contrast, chromosome 6 , with 362 bin markers covering $123.82 \mathrm{cM}$, corresponds to the shortest genetic length covered in this map (Fig. 1, Table 5). The genetic gap length varied from $2.5 \mathrm{cM}$ (on chromosome 6) to $8.22 \mathrm{cM}$ (on chromosome7), and five genetic gaps varied from $5 \mathrm{cM}$ to $9 \mathrm{cM}$ while the other five ranged from $2.5 \mathrm{cM}$ to $4.3 \mathrm{cM}$ (Table 5).

\section{QTLs and genes associated with root related traits at the seedling stage}

The whole maize genome was scanned for root QTLs via inclusive composite interval mapping for additive QTL (ICIM-ADD) with LOD $\geq 2.5$ as the threshold. A total of fourteen (14) substantial QTLs with a phenotypic variance explained, ranging from 1.78 to $16.05 \%$ were obtained across the two time-points (Fig. 2; Table 6). The detected QTLs were allocated to all chromosomes except chromosomes 3, 6, and 9, with no significant QTL detected. Chromosome 4 contained the highest number of QTLs, with 4 QTLs detected (Fig. 2; Table 6). Other chromosomes contained between 1 and 3 QTLs. Different QTL numbers were identified for each time-point, eleven and three at 5 and 15 dag, respectively (Table 6). When examining the number of QTL inheriting parental favorable alleles per time-point, as expected, those alleles did not segregate from the parents uniformly (Table 6). The alleles involved in increasing root morphological characteristics at ten chromosomal positions belonged to the maternal inbred P014. At the same time, the paternal line E1312 contributed to four underlying loci,therefore, the imperative implication of the two parents in root features discrimination. Subsequently, QTL clusters were identified at both timepoints. The four QTLs detected on chromosome $4\left(\mathrm{qPRA}_{5 \mathrm{~d}^{-}}\right.$ 4-1, $\quad$ qSUA $_{5 \mathrm{~d}}-4-1, \quad \mathrm{qSDW}_{15 \mathrm{~d}}-4-1, \quad$ and qTPB $\left._{15 \mathrm{~d}}-4-1\right)$ overlapped and spanned one genetic region, at $90.5-92.5 \mathrm{cM}$ interval (Fig. 2, Table 6). The gene model GRMZM2G068506 predicted to encode a glucose-1phosphate adenylyltransferase was found within this chromosomal region as associated with these four roots above traits (Table 7). All QTLs detected on chromosome 1 


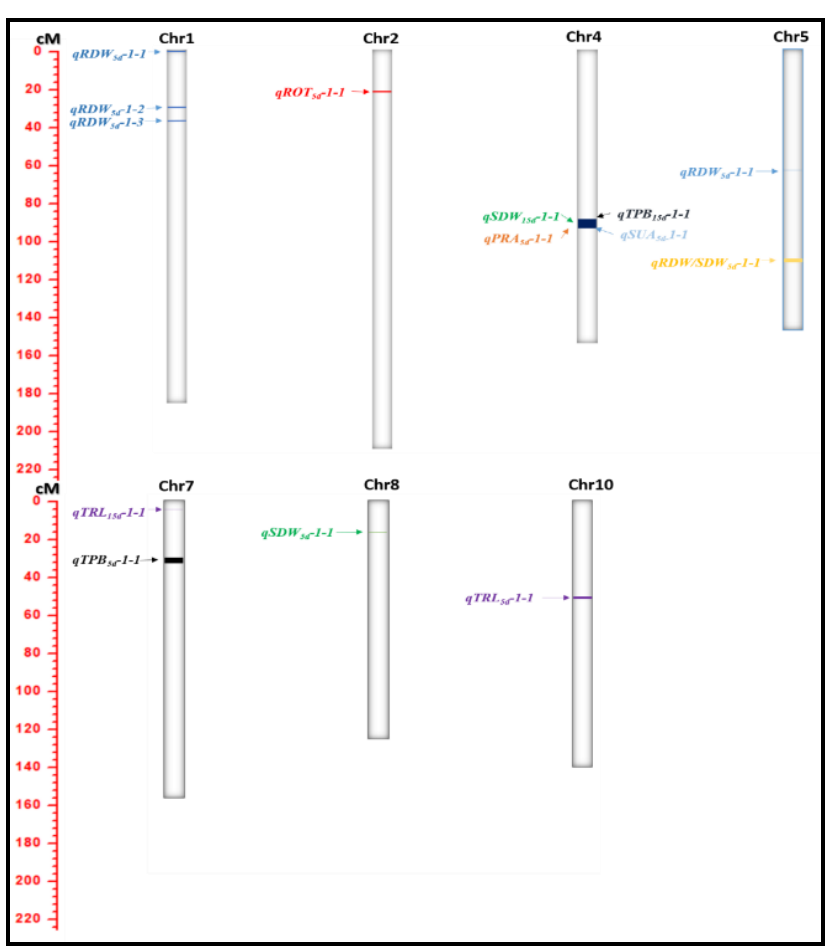

Fig. 2: Maize GBS-SNPs map showing QTLs for root dry weight (RDW), shoot dry weight (SDW), total plant biomass(TPB), root to shoot dry weight (RDW/SDW), total length of roots (TRL), projected area (PRA), root surface area (SUA) and root tips (ROT) at 5 and 15 days after germination in the P014 $\times$ E1312 RIL population. Each trait is symbolized by one specific color and the location is relatively proportional to that of the linkage map length expressed in $\mathrm{cM}$

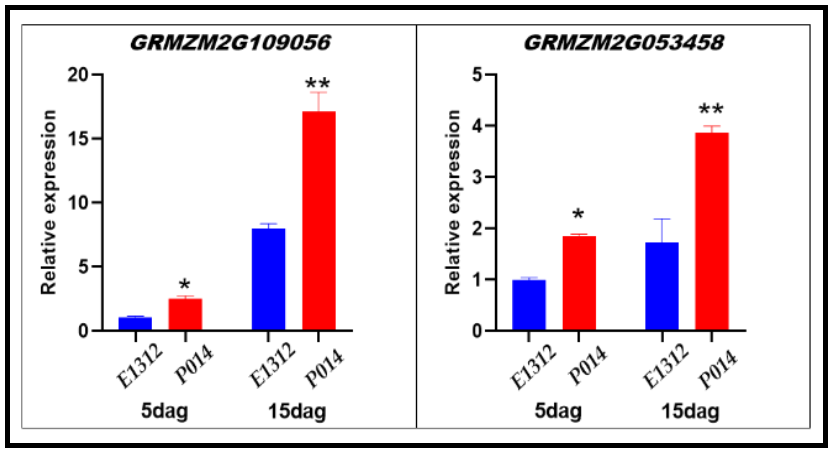

Fig. 3: Relative expression levels of two candidate genes (GRMZM2G109056 and GRMZM2G053458) in the primary roots (sampled at $5 \mathrm{dag}$ and $15 \mathrm{dag}$ ) of the two parental accessions E1312 (in blue) and P014 (in red) tested via $\mathrm{qRT}-\mathrm{PCR}$. * and ** show the significance at $P<0.05$ and $P<0.01$, respectively

are related to $\mathrm{RDW}\left(\mathrm{qRDW}_{5 \mathrm{~d}^{-1}} 1-1, \mathrm{qRDW}_{5 \mathrm{~d}^{-1}-2}\right.$, and $\mathrm{qRDW}_{\left.5 \mathrm{~d}^{-1}-3\right)}$ and spanned the $0-35.5 \mathrm{cM}$ chromosomal region with QTL $\left(\mathrm{qRDW}_{5 \mathrm{~d}}-1-2, \mathrm{LOD}=19.39, \mathrm{PVE}=\right.$ $16.05 \%)$ as the most significant QTL detected at 5dag (Table 6). Interestingly, this region harbored five candidate genes viz., GRMZM2G109056, GRMZM2G123159,
GRMZM2G153476, GRMZM5G874478, and GRMZM2G053458 encoding for a lipoxygenase4, a silencing gene B102, a $30 \mathrm{~S}$ ribosomal protein S13 chloroplastic-like, a glycine-rich RNA-binding protein 8, and a ferredoxin 3 protein, respectively (Table 7). At 5 dag, the QTL on chromosome 10 (qTRL $_{5 \mathrm{~d}}-10-1, \mathrm{LOD}=3.46$, $\mathrm{PVE}=8.5 \%$ ) presented the largest additive effect (Table 6). This QTL inherited the favorable alleles from E1312 line. It harbored two candidate genes GRMZM2G116542 and GRMZM2G016477 predicted to encode a putative Spc97 / Spc98 family of spindle pole body (SBP) component and a putative leucine-rich repeat receptor-like protein kinase, respectively (Table 7). At 15dag, the QTL located on chromosome 7 and involved in TRL (qTRL ${ }_{15 \mathrm{~d}^{-}} 7-1, \mathrm{LOD}=$ $62.6, \mathrm{PVE}=1.78 \%$ ) showed the highest effect in increasing root related-traits (Table 6). This QTL inherited the favorable alleles from $\mathrm{P} 014$ line.

Collectively, four QTL clusters on chromosome 4

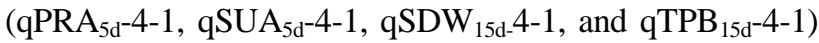
and two closely located QTLs on chromosome $1\left(\mathrm{qRDW}_{5 \mathrm{~d}^{-}}\right.$ 1-2, qRDW $\mathrm{dd}^{-1-3}$ ) were detected in this study (Fig. 2, Table 6). The remaining QTLs were distributed in different chromosomal regions. Further analysis of the QTL results indicated that each co-located QTL position's root characteristics were strongly linked to one another. The details of all candidate genes (27) associated with the different QTLs and the functional annotations are presented in Table 7.

\section{Gene expression in parental lines}

The expression analysis results related to the two evaluated candidate genes GRMZM2G109056 and GRMZM2G053458 showed significant differences in gene expression levels between the two parental materials in primary roots at both $5 \mathrm{dag}$ and $15 \mathrm{dag}(P<0.05, P<0.01$, Fig. 3). As shown in Figure 3, the two genes showed higher expression level for P014 than E1312 at both time-points (5 dag and $15 \mathrm{dag}$ ), which supports the apparent significant phenotypic differences observed among the two parental lines (Table 3 ) as well as the regulating role of the evaluated genes in root growth. Thus, at 5 dag, GRMZM2G109056 and GRMZM2G053458 exhibited approximately 2.5-fold and 1.8-fold higher expression in the P014 line compared to E1312, respectively. Similarly, at 15 dag, GRMZM2G109056 and GRMZM2G053458 showed 2.2fold higher expression in P014 compared to E1312 (Fig. 3).

\section{Discussion}

Wide ranges of variations in terms of root and shoot biomass (RDW, SDW, TPB) amassed were observed between P014 and E1312 at both time-points investigated. The female parent P014 achieved significantly more root tip number (ROT) with higher total root length (TRL). The decrease in ARD may be attributed to the formation over time of new 
Moussa et al. / Intl J Agric Biol, Vol 25, No 4, 2021

Table 5: Properties of the high-density linkage map generated from P014 × E1312

\begin{tabular}{|c|c|c|c|c|c|}
\hline Linkage Group ID & Total Marker & Total Distance (cM) & Average Distance (cM) & Max Gap (cM) & Gap < 5 cM $(\%)$ \\
\hline 1 & 389 & 185.41 & 0.48 & 4.10 & 3.11 \\
\hline 2 & 477 & 209.19 & 0.44 & 4.30 & 0.95 \\
\hline 3 & 453 & 150.54 & 0.33 & 5.62 & 5.18 \\
\hline 5 & 329 & 147.19 & 0.45 & 6.13 & 5.51 \\
\hline 6 & 362 & 123.82 & 0.34 & 2.50 & 3.11 \\
\hline 7 & 635 & 155.62 & 0.25 & 8.22 & 3.02 \\
\hline 8 & 285 & 124.63 & 0.44 & 4.00 & 2.52 \\
\hline 9 & 321 & 124.29 & 0.39 & 5.58 & 5.23 \\
\hline 10 & 296 & 139.12 & 0.47 & 5.53 & 16.44 \\
\hline Total & 4235 & 1514.57 & 0.38 & 8.22 & 4.70 \\
\hline
\end{tabular}

Table 6: Summary of root and shoot traits QTLs detected in P014 $\times$ E1312 RIL population

\begin{tabular}{|c|c|c|c|c|c|c|c|c|}
\hline $\mathrm{QTL}^{\mathrm{a}}$ & Chr & Bin & Peak Pos (cM) & Marker interval & Genetic interval (cM) & LOD score & $\mathrm{PVE}^{\mathrm{b}}(\%)$ & Addc eff. \\
\hline \multicolumn{9}{|l|}{$5 \mathrm{dag}$} \\
\hline $\mathrm{qSDW}_{5 \mathrm{~d}}-8-1$ & 8 & 8.09 & 17 & snp53934-snp53932 & $16.5-19.5$ & 3.01 & 6.18 & 0.01 \\
\hline $\mathrm{qRDW}_{5 \mathrm{~d}^{-1-1}}$ & 1 & 1.11 & 1 & snp9142-snp9174 & $0-1.5$ & 8.17 & 5.95 & -0.01 \\
\hline $\mathrm{qRDW}_{5 \mathrm{~d}}-1-2$ & 1 & 1.09 & 30 & snp8010-snp8064 & $29.5-30.5$ & 19.39 & 16.05 & 0.01 \\
\hline $\mathrm{qRDW}_{5 \mathrm{~d}}-1-3$ & 1 & 1.09 & 35 & snp7954-snp7955 & $34.5-35.5$ & 10.19 & 7.71 & -0.01 \\
\hline $\mathrm{qRDW}_{5 \mathrm{~d}^{-}}-5-1$ & 5 & 5.03 & 110 & snp32110-snp32111 & $107.5-110.5$ & 2.90 & 1.89 & 0.01 \\
\hline $\mathrm{qTPB}_{5 \mathrm{~d}^{-}}-7-1$ & 7 & 7.04-05 & 34 & snp48526-snp48153 & $31.5-38.5$ & 2.61 & 6.26 & 0.01 \\
\hline qRDW/SDW ${ }_{5 d}-5-1$ & 5 & $5.04-05$ & 62 & snp36024-snp35632 & $60.5-62.5$ & 3.38 & 8.37 & -0.22 \\
\hline qTRL $_{5 \mathrm{~d}^{-}} 10-1$ & 10 & $10.05-06$ & 51 & snp62466-snp62578 & $50.5-51.5$ & 3.46 & 8.50 & -7.48 \\
\hline $\mathrm{qPRA}_{5 \mathrm{~d}}-4-1$ & 4 & 4.05 & 92 & snp25434-snp25474 & $90.5-92.5$ & 2.63 & 6.57 & 0.83 \\
\hline $\mathrm{qSUA}_{5 \mathrm{~d}}-4-1$ & 4 & 4.05 & 92 & snp25434-snp25474 & $90.5-92.5$ & 2.63 & 6.58 & 2.61 \\
\hline $\mathrm{qROT}_{5 \mathrm{~d}}-2-1$ & 2 & 2.09 & 23 & snp16526-snp16488 & $22.5-23.5$ & 2.54 & 8.30 & 39.54 \\
\hline \multicolumn{9}{|l|}{$15 \mathrm{dag}$} \\
\hline $\mathrm{qSDW}_{15 \mathrm{~d}}-4-1$ & 4 & 4.05 & 91 & snp25452-snp25434 & $90.5-92.5$ & 3.28 & 8.22 & 0.02 \\
\hline $\mathrm{qTPB}_{15 \mathrm{~d}}-4-1$ & 4 & 4.05 & 91 & snp25452-snp25434 & $90.5-92.5$ & 3.59 & 8.91 & 0.02 \\
\hline qTRL $_{15 d^{-}}-7-1$ & 7 & 7.06 & 4 & snp48875-snp48869 & $3.5-4.5$ & 62.60 & 1.78 & 123.40 \\
\hline
\end{tabular}

Table 7: Candidate genes within the QTL regions and functional annotations

\begin{tabular}{|c|c|c|c|c|}
\hline QTL & Trait & $\mathrm{Chr}$ & Candidate gene & Functional annotation \\
\hline \multirow[t]{3}{*}{$\mathrm{qRDW}_{5 \mathrm{~d}^{-1}}-2 ; \mathrm{qRDW}_{5 \mathrm{~d}^{-1-3}}$} & RDW & 1 & GRMZM2G109056 & lipoxygenase4 \\
\hline & & 1 & GRMZM2G123159 & silencing gene B 102 \\
\hline & & 1 & GRMZM2G153476 & 30S ribosomal protein S13, chloroplastic-like \\
\hline \multirow[t]{2}{*}{$\mathrm{qRDW}_{5 \mathrm{~d}^{-1}}-1$} & RDW & 1 & GRMZM5G874478 & glycine-rich RNA-binding protein 8 \\
\hline & & 1 & GRMZM2G053458 & ferredoxin 3 \\
\hline $\mathrm{qROT}_{5 \mathrm{~d}}-2-1$ & ROT & 2 & GRMZM5G841015 & pollen-specific leucine-rich repeat extensin-like protein 3 \\
\hline $\mathrm{qPRA}_{5 \mathrm{~d}}-4-1 ; \mathrm{qSUA}_{5 \mathrm{~d}}-4-1$ & PRA; SUA & 4 & GRMZM2G068506 & Glucose-1-phosphate adenylyltransferase \\
\hline $\mathrm{qSDW}_{15 \mathrm{~d}}-4-1 ; \mathrm{qTPB}_{15 \mathrm{~d}}-4-1$ & SDW; TPB & 4 & GRMZM2G068506 & \\
\hline \multirow[t]{11}{*}{$\mathrm{qRDW} / \mathrm{SDW}_{5 \mathrm{~d}}-5-1$} & RDW/SDW & 5 & GRMZM2G139300 & cell wall invertase 1 \\
\hline & & 5 & GRMZM2G118737 & Alkaline/neutral invertase CINV2 \\
\hline & & 5 & GRMZM2G326111 & peptidyl-prolyl cis-trans isomerase-like \\
\hline & & 5 & GRMZM2G032628 & amylose extender 1 \\
\hline & & 5 & GRMZM2G143008 & acetolactate synthase 1 \\
\hline & & 5 & GRMZM2G176358 & histone $\mathrm{H} 3.3$ \\
\hline & & 5 & GRMZM2G074017 & ATPase inhibitor \\
\hline & & 5 & GRMZM2G114241 & pentatricopeptide repeat-containing protein At2g29760, chloroplastic \\
\hline & & 5 & GRMZM2G113453 & calmodulin binding protein 1 \\
\hline & & 5 & GRMZM2G337749 & DDT domain-containing protein \\
\hline & & 5 & GRMZM2G064255 & glutathione transferase 17 \\
\hline \multirow[t]{7}{*}{$\mathrm{qTPB}_{5 \mathrm{~d}^{-7}-1}$} & TPB & 7 & GRMZM2G027217 & peroxidase 2-like \\
\hline & & 7 & GRMZM2G054136 & ribosomal proteinS6 \\
\hline & & 7 & GRMZM2G025992 & Superoxide dismutase \\
\hline & & 7 & GRMZM2G056407 & fused leaves 1 \\
\hline & & 7 & GRMZM2G015703 & putative protein kinase superfamily protein \\
\hline & & 7 & GRMZM2G307119 & branched silkless 1 \\
\hline & & 7 & GRMZM2G427815 & peroxidase 3 \\
\hline \multirow[t]{2}{*}{$\mathrm{qTRL}_{5 \mathrm{~d}}-10-1$} & TRL & 10 & GRMZM2G116542 & Spc97 / Spc98 family of spindle pole body (SBP) component \\
\hline & & 10 & GRMZM2G016477 & putative leucine-rich repeat receptor-like protein kinase family protein \\
\hline
\end{tabular}

and fresh roots. Besides, a large spectrum of phenotypic variation was observed from the offspring for most of the evaluated root morphological traits (Table 3 ). Considerable phenotypic variations for root and shoot traits in different maize panels have been previously reported (Pace et al. 2014; Pace et al. 2015a, b; Abdel et al. 2015; Pace et al. 
2015a, b; Song et al. 2016; Ju et al. 2018; Moussa et al. 2018). Broad sense heritability ranged from 7.6 to $71.9 \%$ (Table 3). Similar heritability ranges have been earlier recorded in similar studies regarding maize root traits at various growth stages under field as well as growth chamber conditions (Gu et al. 2017; Sanchez et al. 2018). Due to the high plasticity and quantitative nature of root growth, some root traits were more reproducible than others under the same conditions. In the present study, similar (but with a greater extent at $15 \mathrm{dag}$ ) correlations patterns were detected at all time-points. Therefore, at $15 \mathrm{dag}$, low to strong positive correlations $(r$ between $0.194-0.959, P<0.01)$ were found between root (tips, length, dry weight, surface area, projected area) and shoot dry weight (Table 4). As well, RDW and TRL showed significant positive correlation with all other traits except ARD at 15dag. These findings were consistent with those observed by several authors in similar studies (Song et al. 2016; Hu et al. 2017; Ju et al. 2018). Strong correlation between ROT and RDW confirmed the predominant contribution of root tip number to RDW, TPB and TRL. Recent reports speculated that root system made up of longer but lesser lateral roots are greatly indicated for drought resistance, nitrogen assimilation from soils nitrogendeficient, and increasing final yields under biotic and abiotic constraints than root system composed of shorter lateral roots (Zhan and Lynch 2015; Zhan et al. 2015).

Recent evidence suggests that genetic map quality significantly affects the accuracy of QTL localization (Zhou et al. 2016; Wang et al. 2018). Besides, high marker density maps are more suitable to accurately identify more recombination breakpoints and QTL locations in bi-parental mapping populations. Moreover, it was previously reported that high-density maps could reliably predict potential genes between two successive markers in narrow regions (Zhou et al. 2016). However, most of the root QTL studies in crops have been carried out using relatively low-density marker genetic linkage maps made up with simple sequence repeat (SSR), random amplified polymorphic DNA (RAPD), and restriction fragment length polymorphism (RFLP), resulting consequently in relatively scanty inter-marker intervals. Today, the advances in next-generation sequencing (NGS) technology such as GBS have enabled higher marker densities genetic maps via SNP markers for improved QTLs identification in numerous maize panels for various root morphological traits (Pace et al. 2015a, b; Burton et al. 2015; Zaidi et al. 2016; Sanchez et al. 2018). In this study, a high-quality bin-map composed of 4235 SNPs spanning a genomic distance of $1514.57 \mathrm{cM}$ with an average genetic marker interval of $0.38 \mathrm{cM}$ was constructed (Table 5, Fig. 1). Compared to previous genetic linkage maps using common molecular markers (Mano et al. 2007) as well as GBS-SNPs markers (Burton et al. 2015) in nineteen maize recently root-trait QTL studies (last summary) (Bray and Topp 2018), in which the genetic marker intervals ranged from 0.6 to $17.2 \mathrm{cM}$, the resolution in our study is substantially enhanced. In relatively large populations, QTL mapping based on GBS-SNPs has been lately proposed as an efficient and accurate way to detect rapidly beneficial alleles in genetic resources (Zhou et al. 2016). Mapping of important QTLs involved in root morphological traits is crucial for root and shoot improvement via marker-assisted selection (MAS) and cloning for potential candidate genes controlling root system architecture growth and development in corn (Cai et al. 2012). The availability of fast and reliable root phenotyping techniques through numerous digital imaging softwares and growth media, as well as diverse controlled environment growing systems, offers an efficient and accurate method for fine mapping and cloning root system architecture QTLs. Thus, a considerable number of QTLs underlying root system development in various bi-parental mapping populations have been recently reported (Zurek et al. 2015; Song et al. 2016; Gu et al. 2017; Ju et al. 2018; Bray and Topp 2018). In the present investigation, we detected 14 QTLs controlling the variations of the nine seedling roots related traits evaluated across two different time-points in the P014 × E1312 RIL mapping population. Each QTL explained relatively between 1.78 and $16.05 \%$ of the overall phenotypic variation per trait. Song et al. (2016) recently detected 62 QTLs for 24 seedling root system architecture-related traits using a recombinant inbred line population derived from two Chinese popular commercial inbred lines and also reported small size effects with every single QTL explained from 1.6 to $11.6 \%$ of the total phenotypic variation. Burton et al. $(2014,2015)$ reported similar ranges of phenotypic variation explained, 0.44 to $13.5 \%$ and 4.7 to $12 \%$ using three famous maize US inbred populations in two different studies for various anatomical and architectural root traits, respectively. Compared to those different studies, our findings exhibited somehow higher percentages of phenotypic variance explained.

In this current study, root traits QTLs overlapped or closely linked were localized on chromosomal bins 1.09 , 4.05, and 10.05-10.06 (Table 6). An important number of root related trait QTLs identified are in line with those reported in previous findings. Using the results of fifteen QTL studies from nine different bi-parental mapping populations, a meta-analysis study of QTLs recapitulated many putative QTL clusters related to maize root (Hund et al. 2011). Interestingly, many clusters including Ax-5 (at bin 2.08-2.10), Ax-10 (at bin 5.01-5.03), Rt-11 (at bin 5.03), Ax-11 (at bin 5.04), Ax-16 (at bin 7.04), Rt-14 (at bin 7.04-7.05), and Ax-20 (at bin 10.05-10.06), overlapped with several QTLs $v i z ., \mathrm{qROT}_{5 \mathrm{~d}-2-1}$ (at bin 2.09), $\mathrm{qRDW}_{5 \mathrm{~d}-}$ 5-1 (at bin 5.03), qRDW/SDW ${ }_{5 \mathrm{~d}}-5-1$ (at bin 5.04-5.05), $\mathrm{qTPB}_{5 \mathrm{~d}}-7-1$ (at bin 7.04-7.05), and $\mathrm{qTRL}_{5 \mathrm{~d}}-10-1$ (at bin 10.05-10.06) detected in this current study. Besides, among the twenty-four important MQTLs listed by Hund et al. (2011), four MQTLs namely Rt-11, Ax-11, Ax-16, and Rt14 collocated with three of our detected QTLs, $\mathrm{RRDW}_{5 \mathrm{~d}}-5-1$ (at bin 5.03), qRDW/SDW $5_{5 \mathrm{~d}^{-}}-1$ (at bin 5.04-5.05), and $\mathrm{qTPB}_{5 \mathrm{~d}}-7-1$ (at bin 7.04-7.05)). QTL qRDW/SDW $\mathrm{Sd}^{-5-1}$ 
located on chromosome 5 was closely located near a QTL for total root dry weight to total shoot dry weight ratio identified recently from a widely adapted Chinese hybrid ZD958 (Zheng58 $\times$ Chang7-2) in bin 5.05 (Song et al. 2016). QTL qRDW/SDW ${ }_{5 \mathrm{~d}^{-}}$-1 located on Chromosome 5 and significantly associated with root to shoot dry weight housed the gene models GRMZM2G326111 and GRMZM2G176358 highly expressed in the primary root at VE and V1 stages (Stelpflug et al. 2016; Hoopes et al. 2019). Based on B73, the absolute expression values for GRMZM2G326111 were 41882.26 for the primary roots in VE stage and 34480.2 during V1 developmental stage, relative to 51527.2, which was the maximum expression level (Stelpflug et al. 2016; Hoopes et al. 2019). The expression values for GRMZM2G176358 were 3291.8 at VE stage and 4077.44 at V1 stage, relative to 6319.12, as the maximum expression of GRMZM2G176358 (Stelpflug et al. 2016; Hoopes et al. 2019). QTL qTPB ${ }_{5 \mathrm{~d}}-7-1$ associated with total plant biomass on chromosome 7 harbored the candidate gene GRMZM2G054136 encoding a ribosomal protein S6. Its absolute expression values were 28748.56 at VE and 24409.6 during V1, out of 35599.1, as the maximum expression level of GRMZM2G054136 (Stelpflug et al. 2016; Hoopes et al. 2019). The chromosomal bin 10.05-10.06 region was previously revealed as rich in QTLs for maize root traits $(\mathrm{Gu}$ et al. 2017). In agreement with the involvement of the chromosomal bins 10.05-10.6 (Ax-20) and 2.08-2.10 (Ax$5)$ in the regulation of axile root number and length reported by Hund et al. (2011), we indicate the role of these regions in the control of total root tips number and length $\left(\mathrm{qROT}_{5 \mathrm{~d}^{-}}\right.$ 2-1 and qTRL $\mathrm{d}^{-10-1)}$. Three closely linked loci for root dry

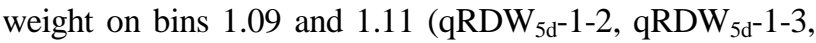
and $\left.\mathrm{qRDW}_{5 \mathrm{~d}}-1-1\right)$ were found in linkage disequilibrium with a QTL detected in a similar study ( $\mathrm{Gu}$ et al. 2017). Interestingly, five genes GRMZM2G109056, GRMZM2G123159, GRMZM2G153476, GRMZM5G874478, and GRMZM2G053458 were remarkably detected within this genetic region. Gene model GRMZM2G109056 codes for a lipoxygenase4 enzyme. Its absolute expression levels were 12832.73 and 18179.96 for the primary root at $\mathrm{VE}$ and $\mathrm{V} 1$, respectively, out of a maximum expression level of 21054.8 (Stelpflug et al. 2016; Hoopes et al. 2019). Gene model GRMZM2G053458 encodes a ferredoxin 3protein with absolute expression levels of 13800.03 at $\mathrm{VE}$ and 27658.8 at $\mathrm{V} 1$, relative to 38759.4, which was the maximum expression level for GRMZM2G053458 (Stelpflug et al. 2016; Hoopes et al. 2019). This putative region might be a special noteworthy locus for marker-assisted breeding critical for further investigations. However, little overlap in QTL detected between previous studies and our work was found for many loci in chromosomal bins $2.09\left(\mathrm{qROT}_{5 \mathrm{~d}^{-}}-2-1\right), 4.05$ (qPRA $_{5 \mathrm{~d}^{-}}$ 4-1, $\quad$ qSUA $_{5 \mathrm{~d}}-4-1, \quad$ qSDW $_{15 \mathrm{~d}}-4-1, \quad$ qTPB $\left._{15 \mathrm{~d}}-4-1\right), \quad 7.06$

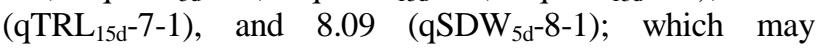
therefore be considered novel. When looking for QTLs overlapping previously recorded gene mutants involved in maize roots development, QTL $\mathrm{qRDW}_{5 \mathrm{~d}}-1-3$ detected for RDW in bin 1.09 at the physical position 263,312,877$263,312,878 \mathrm{bp}$ is positioned just a little more $9 \mathrm{Mb}$ from Rth1, a maize roothairless gene encoding a homolog of sec3 involved in polar exocytosis (Wen et al. 2005). As well, Rth2, another gene regulating maize root hair elongation (Wen and Schnable 1994) collocated with our detected QTL for root to shoot dry weight (qRDW/SDW $\left.{ }_{5 \mathrm{~d}^{-}}-5-1\right)$ in bin 5.04 on chromosome 5.

Quantitative real-time PCR was widely used to validate gene expression with high accuracy and sensitivity (Bustin 2002; Bustin and Nolan 2004). In this study, two potential genes were checked for expression levels using primary root samples from the two parental maize accessions. At all considered time-points (5 dag, $15 \mathrm{dag}$ ), the two evaluated genes GRMZM2G109056 and GRMZM2G053458 acted as positive regulators for root growth with significantly higher expression levels in P014 as compared to E1312. The genes being assessed could be of great value for future investigations.

\section{Conclusion}

This study provides a comprehensive analysis of the genetic architecture of maize root related traits. We performed a QTL mapping using a high-density marker genetic map through 179 maize accessions genotyped by GBS-seq for root morphological traits at two vegetative time-points (5 dag and $15 \mathrm{dag}$ ) under standard laboratory conditions. We identified a total of 14 QTLs using the inclusive composite interval mapping method. The detected QTLs showed individual phenotypic contribution rates ranging from 1.78 to $16.05 \%$ and were significantly associated with SDW (2), RDW (4), TPB (2), RDW/SDW (1), SUA (1), PRA (1), ROT (1), and TRL (2). However, one major QTL region on chromosome 4 was found to be associated with multiple traits, including PRA, SUA, SDW, and TPB (qPRA ${ }_{5 \mathrm{~d}}-4-1$,

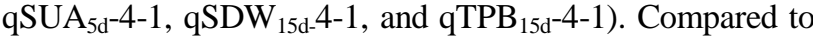
several root QTL previous studies, QTLs located in

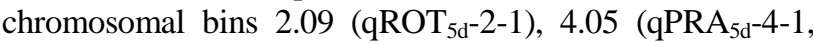
qSUA $\left._{5 \mathrm{~d}}-4-1, \mathrm{qSDW}_{15 \mathrm{~d}}-4-1, \mathrm{qTPB}_{15 \mathrm{~d}}-4-1\right), 7.06$ (qTRL $_{15 \mathrm{~d}^{-}}$$1)$, and $8.09\left(\mathrm{qSDW}_{5 \mathrm{~d}}-8-1\right)$ were found to be novel. Furthermore, two candidate genes GRMZM2G109056 and GRMZM2G053458, overlapping with QTLs for root dry weight on chromosome 1 , were detected and verified for expression level. The evaluated genes were shown to serve as positive regulators for root growth in the mapping parents. Upcoming research will deeply explore these genomic regions to fully illuminate the genetic basis regulating root traits in maize.

\section{Acknowledgments}

This research was funded by the Modern Crop Seed Industry development of Jilin Province, China, (to Piwu Wang). 


\section{Author Contributions}

AAM, PW, and JQ planned the experiments; AAM, AM, $\mathrm{YJ}$, and QZ performed the phenotyping and analyzed the data; AAM prepared the original draft; MGAE, AS, and GA reviewed and edited the manuscript.

\section{Conflict of Interest}

There is no conflict of interest among the authors and institutions where the research has been conducted

\section{Data Availability Declaration}

Data included in this paper are in the custody of corresponding author and can be shared on request

\section{References}

Abdel-Ghani AH, G Bharath, K Jordon, P Constantin, PJ Gonzalez, P Jenaro, R Matamoros, J Pablo, S Martin, M Lee, T Lübberstedt, J Pace (2015). Association analysis of genes involved in maize (Zea mays $\mathrm{L}$.) root development with seedling and agronomic traits under contrasting nitrogen levels. Plant Mol Biol 88:133-147

Abdel-Ghani AH, B Kumar, J Reyes-Matamoros, PJ Gonzalez-Portilla, C Jansen, SMJ Pablo, M Lee, T Lübberstedt (2013). Genotypic variation and relationships between seedling and adult plant traits in maize (Zea mays L.) inbred lines grown under contrasting nitrogen levels. Euphytica 189:123-133

Bray AL, CN Topp (2018). The Quantitative Genetic Control of Root Architecture in Maize. Plant Cell Physiol 59:1919-1930

Burton AL, J Johnson, J Foerster, MT Hanlon, SM Kaeppler, JP Lynch, KM Brown (2015). QTL mapping and phenotypic variation of root anatomical traits in maize (Zea mays L.). Theor Appl Genet 128:93-106

Burton AL, JM Johnson, JM Foerster, CN Hirsch, C Buell, MT Hanlon, SM Kaeppler, KM Brown, JP Lynch (2014). QTL mapping and phenotypic variation for root architectural traits in maize (Zea mays L.). Theor Appl Genet 127:2293-2311

Bustin SA (2002). INVITED REVIEW Quantification of mRNA using realtime reverse transcription PCR (RT-PCR): Trends and problems. $J$ Mol Endocrinol 29:23-39

Bustin SA, T Nolan (2004). Pitfalls of quantitative real-time reversetranscription polymerase chain reaction. $J$ Biomol Technol 15:155-166

Cai H, F Chen, G Mi, F Zhang, HP Maurer, W Liu, JC Reif, L Yuan (2012). Mapping QTLs for root system architecture of maize (Zea mays L.) in the field at different developmental stages. Theor Appl Genet 125:1313-1324

Das A, H Schneider, J Burridge, AKM Ascanio, T Wojciechowski, CN Topp, JP Lynch, JS Weitz, A Bucksch (2015). Digital imaging of root traits (DIRT): A high-throughput computing and collaboration platform for field-based root phenomics. Plant Meth 11:51-62

Galkovskyi T, Y Mileyko, A Bucksch, B Moore, O Symonova, CA Price, CN Topp, AS Iyer-Pascuzzi, PR Zurek, S Fang, J Harer, PN Benfey, JS Weitz (2012). GiA Roots: Software for the high throughput analysis of plant root system architecture. BMC Plant Biol 12; Article 116

Gifford ML, JA Banta, MS Katari, J Hulsmans, L Chen, D Ristova, D Tranchina, MD Purugganan, GM Coruzzi, KD Birnbaum (2013). Plasticity regulators modulate specific root traits in discrete nitrogen environments. PLoS Genet 9; Article e1003760

Gu D, X Mei, T Yu, N Sun, D Xu, C Liu, Y Cai (2017). QTL identification for brace-root traits of maize in different generations and environments. Crop Sci 57:13-21
Hammer GL, Z Dong, G McLean, A Doherty, C Messina, J Schussler, C Zinselmeier, S Paszkiewicz, M Cooper (2009). Can changes in canopy and/or root system architecture explain historical maize yield trends in the US corn belt? Crop Sci 49:299-312

Hochholdinger F, R Tuberosa (2009). Genetic and genomic dissection of maize root development and architecture. Curr Opin Plant Biol 12:172-177

Hoopes GM, JP Hamilton, JC Wood, E Esteban, A Pasha, B Vaillancourt, NJ Provart, CR Buell (2019). An updated gene atlas for maize reveals organ-specific and stress-induced genes. Plant J 97:1154-1167

Hu S, DL Sanchez, C Wang, AE Lipka, Y Yin, CAC Gardner, T Lübberstedt (2017). Brassinosteroid and gibberellin control of seedling traits in maize (Zea mays L.). Plant Sci 263:132-141

Hund A, R Reimer, R Messmer (2011). A consensus map of QTLs controlling the root length of maize. Plant Soil 344:143-158

Iannucci A, D Marone, MA Russo, PD Vita, V Miullo, P Ferragonio, A Blanco, A Gadaleta, AM Mastrangelo (2017). Mapping QTL for root and shoot morphological traits in a Durum Wheat $\times T$. dicoccum segregating population at seedling stage. Intl J Genomics 2017; Article 6876393

Ju C, W Zhang, Y Liu, Y Gao, X Wang, J Yan, X Yang (2018). Genetic analysis of seedling root traits reveals the association of root traits with other agronomic traits in maize. BMC Plant Biol 18; Article 117

Laperche A, F Devienne-Barret, O Maury, JL Gouis, B Ney (2006). A simplified conceptual model of carbon/nitrogen functioning for QTL analysis of winter wheat adaptation to nitrogen deficiency. Theor Appl Genet 113:1131-1146

Li F, H Jia, L Liu, C Zhang, Z Liu, Z Zhang (2014). Quantitative trait loci mapping for kernel row number using chromosome segment substitution lines in maize. Genet Mol Res 13:1707-1716

Liu X, R Li, X Chang, R Jing (2013). Mapping QTLs for seedling root traits in a doubled haploid wheat population under different water regimes. Euphytica 189:51-66

Liu Z, K Gao, S Shan, R Gu, Z Wang, EJ Craft (2017). Comparative analysis of root traits and the associated QTLs for maize seedlings grown in paper roll, hydroponics and vermiculite culture system. Front Plant Sci 8; Article 436

Livak KJ, TD Schmittgen (2001). Analysis of relative gene expression data using real-time quantitative PCR and the $2^{-\triangle \Delta C T}$ method. Methods 25:402-408

Lynch JP (2013). Steep, cheap, and deep: An ideotype to optimize water and $\mathrm{N}$ acquisition by maize root systems. Ann Bot 112:347-357

Mano Y, F Omori, T Takamizo, B Kindiger, RM Bird, C Loaisiga, H Takahashi (2007). QTL mapping of root aerenchyma formation in seedlings of a maize $\times$ rare teosinte Zea nicaraguensis Cross. Plant Soil 295:103-113

Manoli A, A Sturaro, S Trevisan, S Quaggiotti, A Nonis (2012). Evaluation of candidate reference genes for qPCR in maize. $J$ Plant Physio 169:807-815

Meng L, H Li, L Zhang, JJTCJ Wang (2015). QTL IciMapping: Integrated software for genetic linkage map construction and quantitative trait locus mapping in biparental populations. Crop J 3:269-283

Moussa AA, V Salako, DC Gbemavo, M Zaman-Allah, R Kakaï, Y Bakasso (2018). Performances agro-morphologiques des variétés locales et améliorées de maïs au sud-ouest du Niger. Afr Crop Sci J 26:157-173

Pace J, C Gardner, C Romay, B Ganapathysubramanian, T Lübberstedt (2015a). Genome-wide association analysis of seedling root development in maize (Zea mays L.). BMC Genomics 16; Article 47

Pace J, X Yu, L Thomas (2015b). Genomic prediction of seedling root length in maize (Zea mays L.). Plant J 83:903-912

Pace J, N Lee, HS Naik, B Ganapathysubramanian, T Lübberstedt (2014). Analysis of maize (Zea mays L.) seedling roots with the highthroughput image analysis tool ARIA (Automatic Root Image Analysis). PLoS One 9; Article e108255

Pierret A, S Gonkhamdee, C Jourdan, JL Maeght (2013). IJ_Rhizo: An open-source software to measure scanned images of root samples. Plant Soil 373:531-539

Price A, R Tuberosa, SD Dorlodot, B Forster (2007). Root system architecture: Opportunities and constraints for genetic improvement of crops. Trends Plant Sci 12:474-481 
Rellán-Álvarez R, G Lobet, H Lindner, P-L Pradier, J Sebastian, MC Yee, Y Geng, C Trontin, T LaRue, A Schrager-Lavelle, CR Haney, R Nieu, J Maloof, JP Vogel, JR Dinnenny (2015). GLO-Roots: An imaging platform enabling multidimensional characterization of soilgrown root systems. Elife 4; Article e07597

Ren Y, X He, D Liu, J Li, X Zhao, B Li, Y Tong, A Zhang, Z Li (2012). Major quantitative trait loci for seminal root morphology of wheat seedlings. Mol Breed 30:139-148

Rosas U, A Cibrian-Jaramillo, D Ristova, JA Banta, ML Gifford, AH Fan, RW Zhou, GJ Kim, G Krouk, KD Birnbaum (2013). Integration of responses within and across Arabidopsis natural accessions uncovers loci controlling root systems architecture. Proc Natl Acad Sci USA 110:15133-15138

Sanchez DL, S Liu, R Ibrahim, M Blanco, T Lübberstedt (2018). Genome-wide association studies of doubled haploid exotic introgression lines for root system architecture traits in maize (Zea mays L.). Plant Sci 268:30-38

Song W, B Wang, AL Hauck, X Dong, J Li, J Lai (2016). Genetic dissection of maize seedling root system architecture traits using an ultra-high-density bin-map and a recombinant inbred line population. J Integr Plant Biol 58:266-279

Stelpflug SC, RS Sekhon, B Vaillancourt, CN Hirsch, CR Buell, N deLeon, SM Kaeppler (2016). An expanded maize gene expression atlas based on RNA sequencing and its use to explore root development. Plant Genome 9; Article 0025

Symonova O, CN Topp, H Edelsbrunner (2015). DynamicRoots: A software platform for the reconstruction and analysis of growing plant roots. PLoS One 10; Article e 0127657

Wang B, H Liu, Z Liu, X Dong, J Guo, W Li, J Chen, C Gao, Y Zhu, $X$ Zheng (2018). Identification of minor effect QTLs for plant architecture related traits using super high-density genotyping and large recombinant inbred population in maize (Zea mays L.). BMC Plant Biol 18; Article 17

Wang J, H Li, L Zhang, L Meng (2016). User's manual of QTL IciMapping. The Quantitative Genetics Group, Institute of Crop Science, Chinese Academy of Agricultural Sciences (CAAS), Beijing 100081, China, and Genetic Resources Program, International Maize and Wheat Improvement Center (CIMMYT), Apdo Postal 6-641, 06600 Mexico, DF, Mexico
Wasaya A, X Zhang, Q Fang, Z Yan (2018). Root phenotyping for drought tolerance: A review. Agronomy 8; Article 241

Wen T, F Hochholdinger, M Sauer, W Bruce, PS Schnable (2005). The roothairless 1 gene of maize encodes a homolog of sec3, which is involved in polar exocytosis. Plant Physiol 138:1637-1643

Wen T, PS Schnable (1994). Analyses of mutants of three genes that influence root hair development in Zea mays (Gramineae) suggest that root hairs are dispensable. Amer $J$ Bot $81: 833-842$

Wu Y, PR Bhat, TJ Close, S Lonardi (2008). Efficient and accurate construction of genetic linkage maps from the minimum spanning tree of a graph. PLoS Genet 4; Article e1000212

Zaidi P, K Seetharam, G Krishna, L Krishnamurthy, S Gajanan, R Babu, M Zerka, M Vinayan, B Vivek (2016). Genomic regions associated with root traits under drought stress in tropical maize (Zea mays $\mathrm{L}$.). PLoS One 11; Article e0164340

Zhan A, JP Lynch (2015). Reduced frequency of lateral root branching improves $\mathrm{N}$ capture from low-N soils in maize. $J$ Exp Bot 66:2055-2065

Zhan A, H Schneider, JP Lynch (2015). Reduced lateral root branching density improves drought tolerance in maize. Plant Physiol 168:1603-1615

Zhang K, S Liu, W Li, S Liu, X Li, Y Fang, J Zhang, Y Wang, S Xu, J Zhang, J Song, Z Qi, X Tian, Z Tian, W X Li, H Ning (2018). Identification of QTNs controlling seed protein content in soybean using multi-locus genome-wide association studies. Front Plant $\mathrm{Sci}$ 9; Article 1690

Zhou Z, C Zhang, Y Zhou, Z Hao, Z Wang, X Zeng, H Di, M Li, D Zhang, H Yong, S Zhang, J Weng, X Li (2016). Genetic dissection of maize plant architecture with an ultra-high-density bin map based on recombinant inbred lines. BMC Genomics 17; Article 178

Zhu J, SM Kaeppler, JP Lynch (2005). Mapping of QTLs for lateral root branching and length in maize (Zea mays L). under differential phosphorus supply. Theor Appl Genet 111:688-695

Zurek PR, CN Topp, PN Benfey (2015). QTL mapping for maize root system traits. Plant Physiol 167:1487-1496 